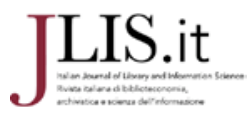

\title{
Le biblioteche di Luigi Crocetti
}

\author{
Tommaso Giordano
}

La differenza "abissale" tra l'autore che ogni anno sforna un libro "e lo studioso intelligente e pudico che pubblica piccoli scritti, risultato di una lunga ricerca è che quest'ultimo apre alla conoscenza mondi altrimenti manipolati e stravolti da quei poligrafi". Queste parole di Giulio Einaudi $(1988,94)$, mi sono tornate in mente più volte nel leggere i saggi, recensioni e paperoles raccolti ne Le biblioteche di Luigi Crocetti (Desideri, Petrucciani 2014).

La prima cosa che mi vien subito da dire del ponderoso volume che oggi presentiamo è che ci troviamo di fronte a un bel libro. Sobria la sua veste editoriale, a cominciare dalla copertina che riporta una foto a colori di Luigi sorridente, in un atteggiamento molto naturale, mentre armeggia con la sua pipa. Ben curato in ogni sua parte, composto con la perizia, la passione e la pazienza di cui sono capaci i due curatori, Laura Desideri e Alberto Petrucciani. Senza dimenticare la rigorosa e particolareggiata cronologia di Silvia Alessandri, che completa il volume. Il tutto ci offre un quadro complessivo della straordinaria molteplicità della produzione di Luigi Crocetti, permettendo di definire in modo più obiettivo e circostanziato il profilo intellettuale e professionale di una personalità che è stata punto di riferimento delle diverse generazioni di bibliotecari degli ultimi cinquanta anni.

JLIS.it Vol. 6, n. 2 (May 2015)

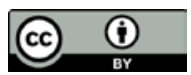


Quando ho saputo del progetto di questo libro, mi sono chiesto se ne valesse la pena, considerato che gli scritti di Crocetti erano stati pubblicati in varie sedi, prima fra tutte il volume Il nuovo il biblioteca (Crocetti 1994) uscito circa venti anni or sono. Le mie perplessità erano originate dalla costatazione che in questa nostra epoca, schiacciata dall'attualità, l'esperienza del passato o la capacità di proiettarsi nel futuro, contano sempre meno. In altri parole, mi chiedevo se gli sviluppi vorticosi di questi ultimi anni, non avessero in qualche modo offuscato anche gli scritti di una personalità del calibro di Luigi Crocetti.

Rileggendo i saggi e le note raccolte il questo libro mi son dovuto ricredere: le mie perplessità erano del tutto infondate e vorrei spiegare perché. Dagli scritti trapela un profilo di Crocetti per alcuni aspetti diverso dall'immagine che molti si erano fatti di lui: cioè, di studioso, dotato di grande equilibrio e moderazione, al di sopra delle parti, che si teneva un po' fuori dalla mischia. Emerge, a tratti, un Crocetti ben più vivace e combattivo (per non dire schierato), persino polemico, ma sempre con il garbo e la sagacia che tutti gli riconoscevano.

Avrei qualche esitazione a classificare il volume sotto la voce biblioteconomia tout court. Si tratta di una messe di riflessioni su biblioteche, archivi e istituzioni analoghe, intesi come strumento di comunicazione culturale e di accesso alla conoscenza, in continua evoluzione, nello sforzo di adeguarsi ai grandi mutamenti tecnologici e sociali in corso. I temi affrontati da Crocetti, pur partendo spesso dalle biblioteche o dai bibliotecari, si pongono in una dimensione culturale che va ben al di là dello specifico professionale. I suoi scritti spaziano dalla biblioteconomia (in tutte le sue sfaccettature), all'archivistica, alla politica culturale, alla letteratura, alla storia. Emerge a tutto tondo il Crocetti intellettuale umanista europeo - se si intende per cultura umanistica un sapere complesso, che unisce valori umani, estetici e scientifici. Un sapere e una sensibilità che si traducono nella capacità di comprendere e 
approfondire la complessità, di interpretare e rendere i chiaroscuri. Ma allo stesso tempo riaffiora l'intellettuale con i piedi per terra, ben consapevole della realtà in cui si muove, che non si lascia andare in astrattezze, eppure capace di volare alto, e anche di manovrare un perfetto atterraggio. Gli scritti, spesso occasionali, prendono spunto da un evento, da "fatti e fatterelli" (per usare un'espressione dell'Autore), da situazioni concrete, per poi proiettarsi in un susseguirsi riflessioni, senza mai perdere il focus, mantenendo il filo del discorso dall'inizio alla fine. A quanto mi consta nessuno dei bibliotecari del Novecento ha saputo registrare con la puntualità di Luigi Crocetti il dibattito sulle biblioteche, avendo come costante quadro di riferimento il fermento politico culturale contemporaneo. Questo, anche in ragione della sua longevità professionale e dei diversi ruoli e molteplici attività in cui era impegnato. Insomma, questi scritti ci forniscono, più di ogni altro documento, i riferimenti per tracciare la storia dell'evoluzione delle biblioteche e della professione bibliotecaria in un lungo arco temporale che vede chiudersi un epoca e aprirsene una nuova.

Più spesso si ha la netta sensazione che le biblioteche o i bibliotecari non siano né l'oggetto, né gli unici destinatari degli scritti di Crocetti. Voglio dire che gli interlocutori cui si rivolge l'Autore prefigurano una audience ben più larga: politici, amministratori, intellettuali, gli utenti stessi delle biblioteche. Prendiamo ad esempio il bellissimo saggio dal titolo La tradizione culturale italiana del Novecento (1999): l'autore parte con una rapida e suggestiva rilettura della storia culturale dell'Italia Unita (tra universalismo e provincialismo) individuando nel policentrismo "la costante e peculiarità della cultura italiana". "Il sistema bibliotecario italiano del passato (di sistema si può parlare solo per metafora) è stato, naturalmente, straordinariamente policentrico, e ha accompagnato e rappresentato degnamente le vicende culturali del territorio". E ancora così? - si chiede Crocetti - "A me sembra che in un passato non più vicinissimo ci sia stata una frattura [...] Quando?" La riforma Gentile (del 1923) "può essere assunta a segno del nefasto cambiamento". 
Con essa i bibliotecari cambiavano status: "da una categoria di alto profilo si passava a una categoria di semplici impiegati: nasceva la burocrazia bibliotecaria" del Novecento. Ma molto peggio "si sarebbe fatto in età democratica, da parte di un altro intellettuale, Giovanni Spadolini". Nel testo del Decreto (Organizzazione del Ministero per i Beni Culturali e Ambientali 1975) "si infieriva sulle biblioteche, non vergognandosi di definirle 'organi periferici del Ministero', ciò che non era stato immaginato neppure ai tempi della dittatura. Se ne ribadiva la burocratizzazione", accentuando il divario tra biblioteconomia e tradizione culturale e contribuendo a svilire la funzione intellettuale dei bibliotecari. "Può fare cultura un organo periferico del ministero?". "La biblioteconomia italiana constata mestamente Crocetti - è ricca di passato e povera di tradizione".

Crocetti è convinto che "le biblioteche debbano per loro natura essere profondamente immerse nel flusso culturale generale". Il rapporto tra intellettuali e biblioteche ritorna spesso nelle pagine di Crocetti: un esempio è il bel saggio dal titolo Le biblioteche di Giorgio Pasquali. (Desideri, Petrucciani 2014) Il tema ricorre anche negli interventi ai convegni "Conservare il Novecento". La cultura appartiene a tutti, ergo le biblioteche non sono un problema esclusivo dei bibliotecari ma un problema di tutti - a cominciare dagli studiosi; dunque tutti ne possono, ne debbono parlare. "Tutti sappiamo - parole di Crocetti - che negli ultimi anni alcuni contributi più intelligenti sul problema delle biblioteche" (Desideri, Petrucciani $2014,190)$. "Qual è l'insegnamento primo che possiamo ricavare da questi contributi, indipendentemente dal loro specifico contenuto critico e propositivo? Nell'averci ricordato/rivelato che i problemi delle biblioteche italiane sono problemi semplici, cioè problemi di funzionamento". "Ma - puntualizza subito dopo - anche "il fatto di funzionare esige una cultura professionale sofisticata". Questa benigna condiscendenza di Crocetti nell'ascoltare seriamente i contributi 'profani', da qualunque parte essi provengano, si trasforma in inusitata durezza quando si tratta di rispondere ad JLIS.it. Vol. 6, n. 2 (May 2015). Art. \#11079 p. 4 
attacchi sprezzanti, ad argomentazioni speciose e approssimative, come un articolo apparso sul Corriere della Sera nel maggio 1986, in cui il giornalista Valerio Riva, dopo aver dileggiato i bibliotecari ( e le bibliotecarie, "patetiche pinzochere"), proponeva di privatizzare le biblioteche "di farne un'agenzia come si usa in America" (sic), di "darle in gestione alle grandi società di informatica". E qui Crocetti depone il fioretto e impugna lo stiletto: allora se è vero come è vero che le biblioteche in tutto il resto del mondo sono in mano ai bibliotecari e funzionano, offriamo a Riva la spiegazione più economica (che certo gli piacerà) [...] i bibliotecari d'altrove sono intelligenti e capaci, gli italiani cretini. E così semplice - continua sarcastico - basterà aprire le frontiere come per i calciatori". Sarebbe invece l'ora che i giornali parlassero di biblioteche permettendo ai lettori di formarsi un'opinione "magari evitando i contributi di chi in biblioteca non si capisce cosa ci vada a fare" (Desideri, Petrucciani 2014, 555-556).

La politica non era la passione di Luigi, neanche nei tempi in cui andava di moda, lui si manteneva a distanza da quel mondo che non gli era congeniale. Questo poteva essere interpretato come atteggiamento ispirato alla cautela, in realtà Crocetti non si sottrae quando il suo ruolo lo richiede. Anzi rileggendo oggi i suoi scritti scopriamo - come ho riferito poc'anzi - un Crocetti più combattivo e graffiante del personaggio pacato che eravamo abituati ad ascoltare. Così nella relazione al congresso di Viareggio (1987): "La definizione di 'occhio vitreo' è la più comune per indicare l'occhio del responsabile politico o amministrativo a colloquio con noi: l'occhio di chi è lontanissimo col pensiero da ciò che gli state dicendo o l'occhio di chi, appunto, non comprende". A quindici anni dall'istituzione delle regioni, Crocetti che pure era schierato a favore del decentramento, come la maggior parte dei bibliotecari, con l'AIB in prima linea, manifesta apertamente la sua "insoddisfazione per i modi con cui l'operazione era stata condotta, anche da parte regionale". Se da una parte l'amministrazione centrale ha messo in opera ogni possibile strumento per mantenere intatte tutte le sue 
prerogative ed è "passata addirittura all'offensiva inaugurando nuove biblioteche 'nazionali' nei luoghi più impensati" - denuncia Crocetti - "il campo dove il lavoro regionale è stato più manchevole quando non assente, è proprio quello per il quale le regioni avevano ricevuto competenze maggiori e sotto molti aspetti esclusive". "Una delle nostre grandi speranze si è andata così affievolendo. Non per questo abbiamo rinunciato ad accenderne un'altra" (Desideri, Petrucciani 2014, 222). Crocetti qui si riferisce a SBN, ben sapendo che la strada è tutta in salita: "le idee di SBN - affermerà in un'altra occasione - volano ben sopra della testa di certi burocrati: pure nelle mani di costoro ci sono doppiette capaci di abbattere le aquile più maestose" (Desideri, Petrucciani 2014, 181).

Un altro aspetto che emerge in tutta evidenza in questi scritti è la straordinaria capacità di comprendere e di interpretare l'evoluzione tecnologica, elemento centrale per capire la trasformazione dei circuiti della conoscenza in questi anni. Crocetti fu tra i pochissimi bibliotecari ad aver posto nei giusti termini la questione fin dal primo momento, ad aver dato un senso all'impiego delle tecnologie nelle biblioteche, a vedere nelle tecnologie un potente mezzo per fare le biblioteche partecipi, anzi elementi attivi nel flusso della comunicazione culturale e scientifica (si veda la relazione introduttiva al congresso di Giardini Naxos del 1981) (Associazione Italiana Biblioteche 1983). Biblioteche e cambiamento è uno dei temi su cui Crocetti ritorna più volte. Nel 1985 pronostica una "metamorfosi della biblioteca", provocata appunto dall'incontro tra biblioteca e tecnologia. Ma il nuovo di cui parla l'Autore non nasce nelle biblioteche, e parlare di "adeguamento [alle tecnologie] non è sbagliato ma è certo molto riduttivo". "Chi si sta adeguando avverte Crocetti - non sono le biblioteche ma l'intera nostra società. Vorrà dire che sono le biblioteche ad approssimarsi alla fine? Il sospetto è autorizzato" (Associazione Italiana Biblioteche 1983, 27). Crocetti pone il problema della quantità e qualità dell'informazione, e solleva due punti di riflessione: il primo è la cooperazione a tutto campo, il secondo è la selezione delle fonti (cioè, la questione della JLIS.it. Vol. 6, n. 2 (May 2015). Art. \#11079 p. 6 
qualità dei contenuti e della responsabilità intellettuale dei bibliotecari). Queste riflessioni sono del 1985. Più tardi ritornerà ancora sul tema con straordinaria lucidità profetica. Nel 2001, al convegno Conservare il Novecento (Zagra 2012), riferendosi agli archivi personali: "Quella del Novecento sarà con ogni probabilità l'ultima cultura a poter essere documentata nei modi tutto sommato classici [...] non ci attendiamo certo la morte del libro o della carta stampata; ma proprio nel campo di cui stiamo parlando, la morte di quell'apparato di contorno fatto di schede, di foglietti di appunti schizzi, sgorbi, cancellature. Appunto, in una parola, scartafacci e paperoles [...] Gli archivi del futuro, di che cosa saranno fatti? Biblioteconomia e archivistica annaspano ancora e forse annasperanno per molto tempo". Poi commenta: "Naturalmente è comodo rimandare le soluzioni all'avvenire" - e aggiunge con un pizzico di malizia: "forse la stessa tecnologia con il suo potere autorisarcente troverà $\mathrm{i}$ rimedi, ma sarà utile cominciare a pensarci fin d'ora con riflessioni e ipotesi" (Desideri, Petrucciani 2014, 28).

In questi ultimi mesi si parla di riforma del Ministero dei beni culturali e di una discutibile riorganizzazione delle biblioteche e degli archivi statali: mi sono chiesto che cosa ne avrebbe detto Luigi Crocetti. Luigi diffidava delle grandi riforme imposte dall'alto, guardava con sospetto a quelle che lui definiva con disprezzo "operazione di ingegneria burocratica", che si traducono spesso in manovre di facciata: cambiare tutto per non cambiare niente, salvo sottrarre alle biblioteche le già troppo esigue risorse. Crocetti avversava profondamente le manovre verticistiche, tanto più se venivano perseguite al di là di qualsiasi criterio scientifico e professionale. La biblioteconomia di Crocetti era una biblioteconomia innovativa, di grande progettualità, di orizzonte internazionale, ma estremamente rispettosa della tradizione culturale e del patrimonio esistente. Potremmo paragonare la biblioteconomia di Crocetti all'architettura di Renzo Piano: vale a dire, una disciplina in cui si fondono cultura, scienza e bisogni della comunità. Progettare il nuovo non vuole dire rottamare quello che 
c'è, ma integrare il nuovo nel bello esistente. Renzo Piano parla di "rammendo", quando si tratta di intervenire in situazioni complesse come i centri storici o le periferie delle città. Non so se Luigi abbia usato in qualche occasione la parola "rammendo", ma la vedo molto congeniale al suo modo di vedere le cose. Oggi le biblioteche italiane si trovano di fronte a una sfida che assomiglia in qualche modo alla battaglia per la conservazione dei centri storici combattuta negli anni Sessanta, con la differenza (purtroppo) che non c'è la diffusa consapevolezza politica e culturale di allora.

Vorrei, infine, richiamare un altro aspetto straordinario degli scritti di Crocetti: lo stile. Crocetti è uno scrittore elegante, colto, ma mai saccente. Usa uno stile semplice, con scarso ricorso ai termini tecnici, in modo che possano capire gli addetti come i non addetti. Alcuni saggi, come Il silenzio della biblioteca, oppure Felis bibliotecarum, sono prove di raffinata e godibile letteratura. A volte l'Autore fa appena trasparire il suo humor, altre volte non riesce a trattenere il graffio polemico. Si veda l'inizio tranchant della lettera al Corriere della sera, sopra citata: "Il falso ha un vantaggio sulla verità: che ha bisogno di meno spazio. [...] Per rettificare gli errori in cui si è cacciato Valerio Riva non basterebbe un'intera pagina". Poi ci sono le agili recensioni e le deliziose paperoles, che consiglio di leggere dall'inizio alla fine. Lancio un'idea: perché non estrarre questa parte più godibile degli scritti di Crocetti e farne un volumetto per un pubblico più vasto? In fondo come diceva lui, la cultura e le biblioteche sono di tutti.

Concludo con una frase di Luigi Crocetti, che è un messaggio di saggezza e di speranza, particolarmente adatta ai tempi che stiamo vivendo: "Dalla metamorfosi nascerà una nuova vitalità della biblioteca, se i bibliotecari avranno coraggio di viverla, questa biblioteca, anche se forse non è la biblioteca sognata; di vivere questo mondo anche se non è il migliore dei mondi possibili" (Desideri, Petrucciani 2014, 28). 


\section{Bibliografia}

Associazione Italiana Biblioteche. 1983. Ruolo e formazione del Bibliotecario: Atti del XXIX Congresso dell'Associazione Italiana Biblioteche. Firenze, 29 gennaio-1 febbraio 1981. Firenze: Giunta regionale toscana.

Crocetti, Luigi. 1994. Il nuovo in biblioteca e altri scritti. Roma: Associazione Italiana Biblioteche.

- - - . 1999. "La tradizione culturale italiana del Novecento." In

Associazione Italiana Biblioteche

http://www.aib.it/aib/sezioni/veneto/crocetti.htm.

Le Biblioteche di Luigi Crocetti. Saggi, recensioni, paperoles. 2014. A cura di Laura Desideri e Alberto Petrucciani. Roma: Associazione Italiana Biblioteche.

Einaudi, Giulio. 1988. Frammenti di memoria. Bologna: Rizzoli.

Organizzazione del Ministero per i Beni Culturali e Ambientali. 1975. Conservare il Novecento: carte e libri in vetrina. Convegno, Ferrara, Salone Internazionale dell'arte, del restauro e della conservazione dei beni culturali e ambientali, 1 aprile 2011. 2012. A cura di Giuliana Zagra. Roma: Associazione Italiana Biblioteche. 
T. Giordano, Le biblioteche...

GIORDANO, TOMMASO. tommaso.giordano@eui.eu.

Giordano, Tommaso. "Le biblioteche di Luigi Crocetti". JLIS.it. Vol. 6, n. 2 (May 2015): Art: \#11079. DOI: 10.4403/jlis.it-11079.

ABSTRACT: This article is the text read at the presentation of the book Le Biblioteche di Luigi Crocetti, saggi, recensioni, paperoles (The Libraries of Luigi Crocetti, essays, reviews, paperoles), AIB, 2014, edited by Laura Desideri and Alberto Petrucciani. Luigi Crocetti (died in 2007) was one of the most eminent personalities in the Italian library world of the last fifty years. Some of the most significant milestones of his career are: Director of the Laboratory of Restoration at The National Central Library in Florence, after the flood of the Arno river in 1966; Director of the Library and Archives Department of the Tuscany regional government; President of the Italian Library Association (AIB), Director of the Gabinetto G.P. Vieusseux. Crocetti was also leader of, or influential partner in, the major projects and programs developed in Italy during his professional life, including the DDC 20 Italian Edition, the project SBN (the Italian Library Network), Editor of the journal Biblioteche Oggi, as well as collaborator in various journals and publishing activities. The numerous writings of Luigi Crocetti, published throughout his professional life, register punctually the evolution of libraries and librarian profession in times of major technological, cultural and social changes. Nevertheless Crocetti's writings are not only an historical record of a perceptive (and committed) observer; they also constitute a collection of profound reflections and inspiring input for further discussion on the role of libraries and of culture in the society. Gifted with uncommon erudition, Crocetti shows an extraordinary ability to interpret the current reality and for see the future with a remarkable farsightedness. His writings touch on all aspects of the profession, from book preservation and restoration to public libraries, from cataloguing and indexing to library cooperation and networking, from national libraries, archives and 
museums, to user services. It is quite evident that Crocetti is aiming to an audience far beyond librarians, to include policy makers, managers, intellectuals and all those who have contact with libraries; in a final analysis, he addresses to all citizens, being convinced that "culture is for everyone" and that "libraries must by their nature be deeply immersed in the overall cultural flow". Innovation and renovation of the professional mentality are recurrent themes in the writings of Crocetti. The Author - fully aware of the international debate and attentive to technological change - identified in the progressive loss of contact between libraries and cultural tradition one of the weak points of the Italian librarianship. According to his view, this situation emphasized the 'bureaucratization' of libraries and librarians, which is a major cause of the erosion of their intellectual function. "The Italian librarianship - notes bitterly Crocetti - is rich in the past and poor in tradition". It is a controversial issue, like many others (too often passed over by the professional debate) highlighted by Crocetti in his writing with clarity and equilibrium, like the true intellectual he was, able to rise above the interests of their own sector, but also capable of representing the aspiration to renew the librarian profession in the global dimension of our times.

KEYWORDS: Crocetti, Luigi; librarian profession.

Submitted: 2015-01-03

Accepted: 2015-02-10

Published: 2015-05-15

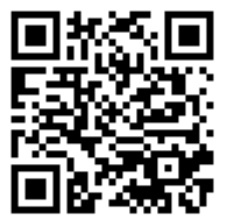

